Musées, Patrimoine et Culture scientifiques et techniques

$155 \mid 2014$

septembre-octobre 2014

\title{
Le parcours : drôle de temps pour une rencontre
}

Joëlle Le Marec

URL : http://journals.openedition.org/ocim/1435

DOI : $10.4000 /$ ocim. 1435

ISSN : 2108-646X

Éditeur

OCIM

Édition imprimée

Date de publication : 1 septembre 2014

Pagination : 5-9

ISSN : 0994-1908

Référence électronique

Joëlle Le Marec, «Le parcours : drôle de temps pour une rencontre », La Lettre de I'OCIM [En ligne],

155 | 2014, mis en ligne le 01 septembre 2016, consulté le 30 avril 2019. URL : http://

journals.openedition.org/ocim/1435; DOI : 10.4000/ocim.1435

Ce document a été généré automatiquement le 30 avril 2019

Tous droits réservés 


\title{
Le parcours : drôle de temps pour une rencontre
}

\author{
Joëlle Le Marec
}

Parcours au Rheinisches Landesmuseum à Bonn (Allemagne).

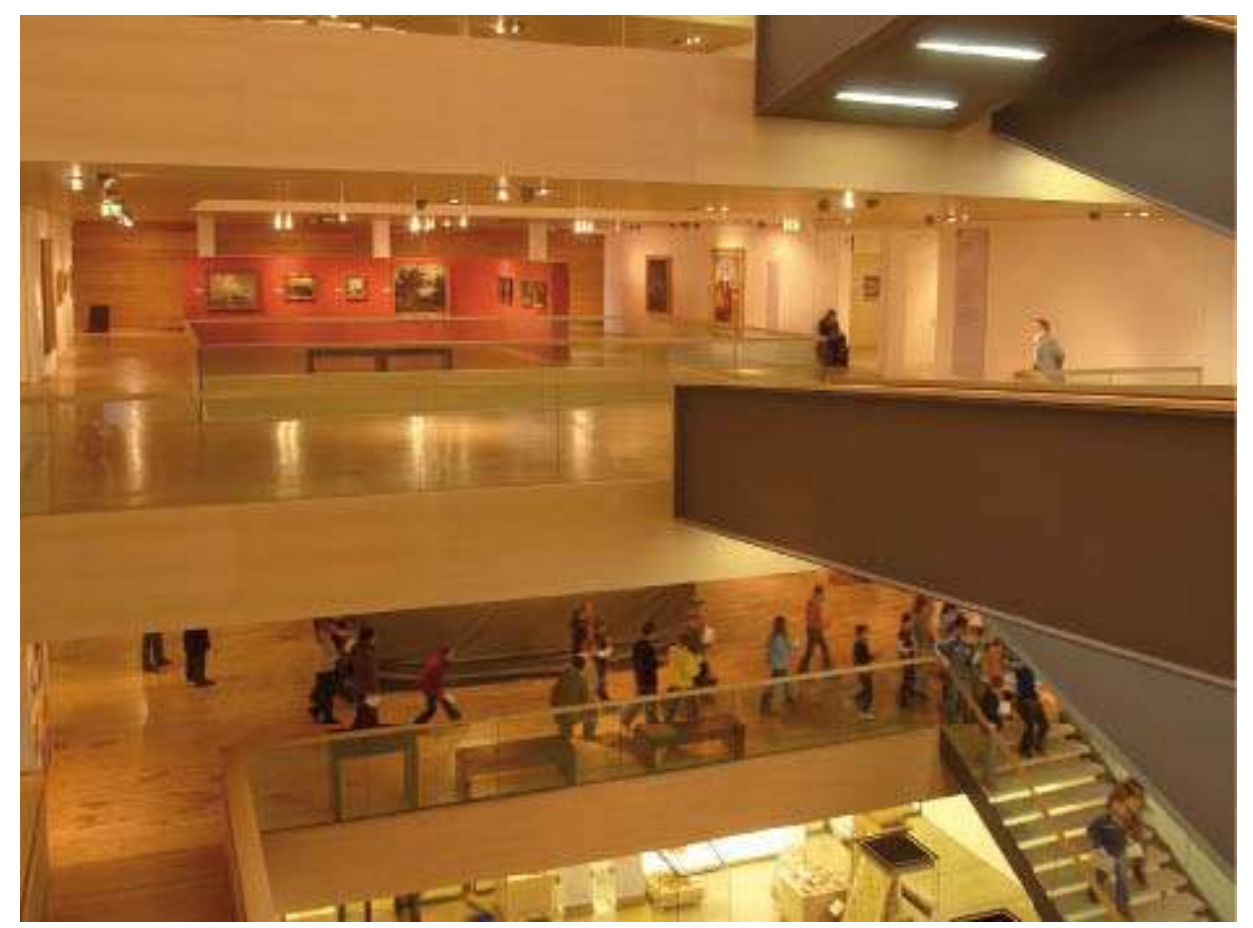

(C) Daniel Schmitt

1 Certaines notions s'élaborent et s'enrichissent dans le fil continu de pratiques, d'interactions, de lectures, d'expériences, et elles exigent une attention nécessaire au point de vue d'autrui. De ce fait, elles sont à la fois très complexes à énoncer verbalement, et relativement simples malgré tout à comprendre dans l'action ou dans l'échange. 
C'est le cas du parcours en muséologie ${ }^{1}$. Il ne s'agit pas d'un concept théorique (comme le langage ou le discours) ni d'un objet empirique simple (comme un panneau ou une conférence) mais d'une notion qui a l'avantage de faire sens pour ceux qui conçoivent et fabriquent des expositions et des dispositifs de médiation muséale, pour ceux qui les animent et les font vivre, pour ceux qui les visitent et pour ceux qui les observent et les étudient. C'est pourquoi le parcours est avant tout considéré ici comme un objet du dialogue, nécessaire à la création et à la réflexion, entre des muséologues, des scénographes, des médiateurs, des visiteurs et des chercheurs.

Ce dialogue permet de se détacher d'une vision mécaniste et fonctionnelle de l'exposition considérée comme résultat d'une chaîne linéaire de tâches successives et fragmentées aboutissant à une production fermée. Il permet également de dépasser l'idée, erronée, que la relation au visiteur serait du type offre/demande (on lui fournit un produit à consommer) ou émission/réception (on élabore un message à destination d'une cible). Les recherches muséales montrent de fait que les choses se passent tout autrement, dans la conception et dans les visites, telles qu'elles sont pratiquées directement.

\section{Le parcours : lieu commun et plateforme d'échange}

Le parcours est donc un foyer de malentendus possibles si les visions divergentes restent implicites mais un domaine de partages possibles très importants entre chercheurs et praticiens, si elles sont explicitées, respectées et débattues. Il permet de penser ensemble :

- dans le cas des concepteurs et médiateurs : des pratiques professionnelles destinées à faire quelque chose pour et avec autrui ;

- dans le cas des visiteurs : des pratiques culturelles fondées sur des propositions qui leur sont adressées ou qui les concernent ;

- dans le cas des chercheurs: des pratiques destinées à comprendre ce qui se passe dans l'exposition.

En effet, nous avons besoin de dire et de représenter des manières de travailler collectivement qui sortent radicalement de l'étau des modèles issus du management de l'activité et de la gestion analytique. Ceux-ci sont certes très convaincants pour les administrateurs, mais ils ne mobilisent guère la créativité propre à des activités qui ne trouvent leur sens que dans une volonté de faire avec autrui (les membres d'une équipe, les partenaires, les représentants d'une population ou du public) et pour autrui (les visiteurs). Ces manières de travailler correspondent à un type de professionnalisme sensible, dynamique, qui assume la complexité inhérente à chaque proposition et expérience. Il nous semble significatif que la muséologie n'ait guère inspiré le type de procédure standardisée de fabrication d'exposition qui pourrait apparaître avec la prolifération des projets. Les muséologues qui ont une très forte expérience de la conception d'exposition se fabriquent eux-mêmes, comme des maîtres artisans, leurs propres outils conceptuels inventés pour formaliser ce travail collectif autour du projet d'exposition ${ }^{2}$. Ces représentations des liens et de toutes les procédures de traduction (entre un propos et un regroupement d'artéfacts par exemple) participent ainsi à la construction d'un discours polyphonique.

6 Le parcours a l'avantage d'être une sorte de plateforme d'échange, à la fois technique et poétique, entre scénographes, muséologues, médiateurs, visiteurs : c'est pourquoi il n'est 
surtout pas besoin de définitions standardisées ni de catégories hiérarchisées pour en traiter. Il facilite des opérations de multiples traductions plus qu'il n'homogénéise les interventions.

7 Ces opérations de traductions sont elles-mêmes des paris qui sont fait en permanence. Elles nécessitent de s'en remettre à l'effort, l'indulgence et l'apport d'autrui. Pratiques de conception et de médiation, pratiques de visite et pratiques de recherche, mobilisent en effet des compétences et des savoir-faire culturels et techniques, mais aussi des questions, des doutes, des désirs sur l'intercompréhension possible avec des interlocuteurs. Et c'est là toute la force et la qualité de ce qui est partagé en fin de compte.

8 Ce que l'on entend par "parcours" n'est pas la même chose selon que l'on est visiteur, scénographe ou médiateur. Il existe des parcours objectivés sur des plans et des parcours vécus, au sens de trajectoires temporelles ou de choix ordonnés et séquencés, dans une multiplicité d'options. En outre on peut penser des parcours d'accès et des parcours de visite, des parcours dans l'exposition et des parcours à toutes les étapes de la conception, des parcours-enfants, des parcours spécifiques pour les visiteurs, des parcours thématiques. On peut aussi penser à une signalétique dans le permanent pour signaler des œuvres particulières. Interviennent également les signalétiques de médiation, les parcours transformés ou créés par la médiation, et même la médiation des parcours, des parcours audioguidés et des visites "augmentées", des parcours urbains et des démarches d'interprétation...

9 Mais le fait qu'il existe des ambiguïtés multiples, parfois antagonistes, entre ces visions n'est nullement gênant : au contraire, cela oblige à dépasser l'idée que le parcours serait avant tout l'aménagement d'une trajectoire dans un espace pour passer d'un endroit à un autre, trajectoire éventuellement enrichie d'autres propositions de médiations.

\section{En commençant par la visite...}




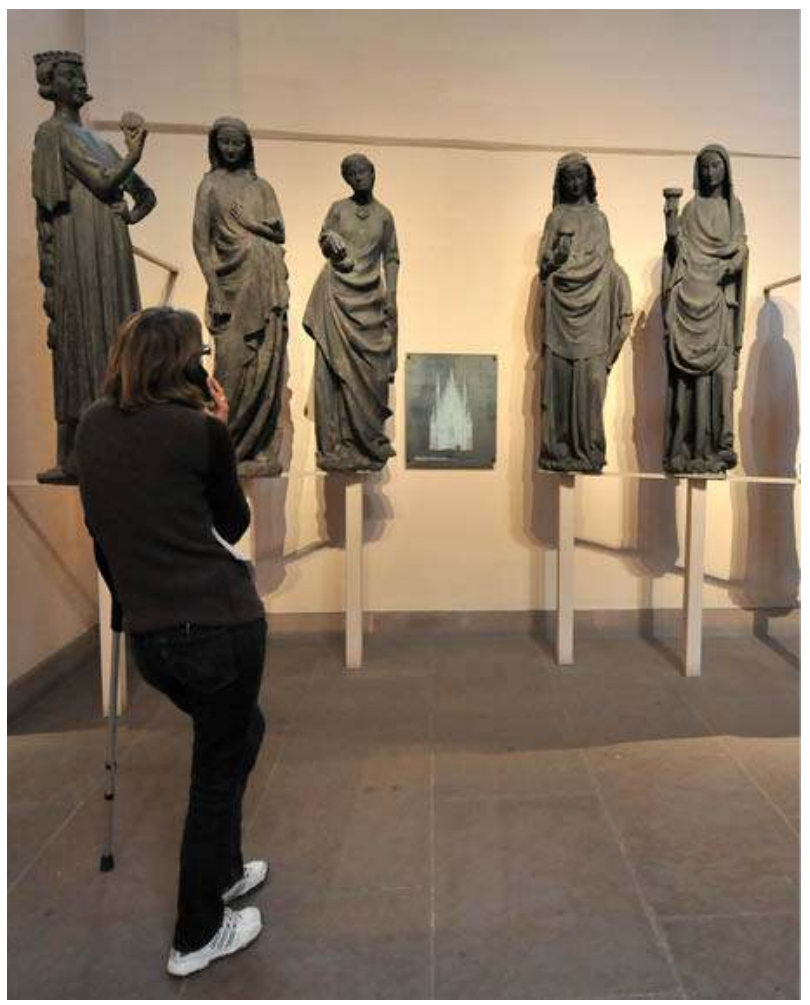

(c) Daniel Schmitt

Au départ de ma réflexion sur le parcours, il y a la découverte des pratiques de visite à travers un très grand nombre d'études de publics, et surtout de la manière dont les visiteurs se sentent guidés par tous ceux qui ont prévu quelque chose à leur intention et qui ont laissé des clés, des indices, des aides pour leur permettre de faire au mieux. L'effort d'attention aux visiteurs peut se nourrir des résultats désormais acquis, robustes de quantités d'évaluations et d'études effectuées par des collègues et par moi-même depuis plusieurs décennies. Il nous semble impossible que des scénographes ignorent encore ces résultats, tant ils sont décisifs et nous apprennent des choses essentielles.

Avant tout, les études font apparaître des pratiques et des états qui débordent très largement la vérification d'un effet ou d'un impact auprès des visiteurs (comme par exemple la seule question "sont-ils satisfaits ?"). Prendre en compte ces études revient à se laisser porter par ce qui, en eux, excède une procédure d'optimisation technique pour se laisser toucher, transformer, inspirer par le contact avec les visiteurs tout comme ils se laissent si souvent toucher et transformer eux-mêmes par les expositions.

En effet, le visiteur se situe dans un espace intentionnel, il le sait et s'appuie sur cette communication avec ceux qui sont absents : il se dit "tout ce qui est ici est là pour moi". Mais cet espace ne s'apparente pas tant à un paysage (une scénographie), plutôt à un chemin, un sentier. L'exposition constitue à ses yeux avant tout une durée, un temps de visite. Par exemple, les visiteurs n'abordent absolument pas de la même manière le début et la fin d'un parcours. Ils se préparent, ils appréhendent parfois, ils découvrent, ils ressentent de la lassitude, de la surprise, ils sont concentrés, émus, ils rencontrent des difficultés, ils passent, ils évitent, hésitent, choisissent, décident, ils attendent et 
s'attendent, ils reviennent, ils prolongent bien après la sortie, puis déjà, au moment des enquêtes, ils synthétisent, travaillent un récit qui souligne les reliefs de l'expérience.

La visite est donc une trajectoire temporelle. Les visiteurs cherchent à l'accomplir au mieux et pour cela, ils se prêtent à ce qui a été prévu pour les guider dans ce qui est avant tout un agencement intentionnel de quantité d'objets et d'informations. On ne le dira jamais assez: le discours se vit et se déploie à travers l'engagement dans la visite. Les métaphores fort allusives de certaines scénographies comptent bien peu, bien moins, que des implications directes (à un carrefour, face à une manipulation) ou des rencontres, lorsque l'on est interpelé, par exemple dans le face à face avec un texte.

Les dispositifs interactifs pour enfants au Vaisseau à Strasbourg
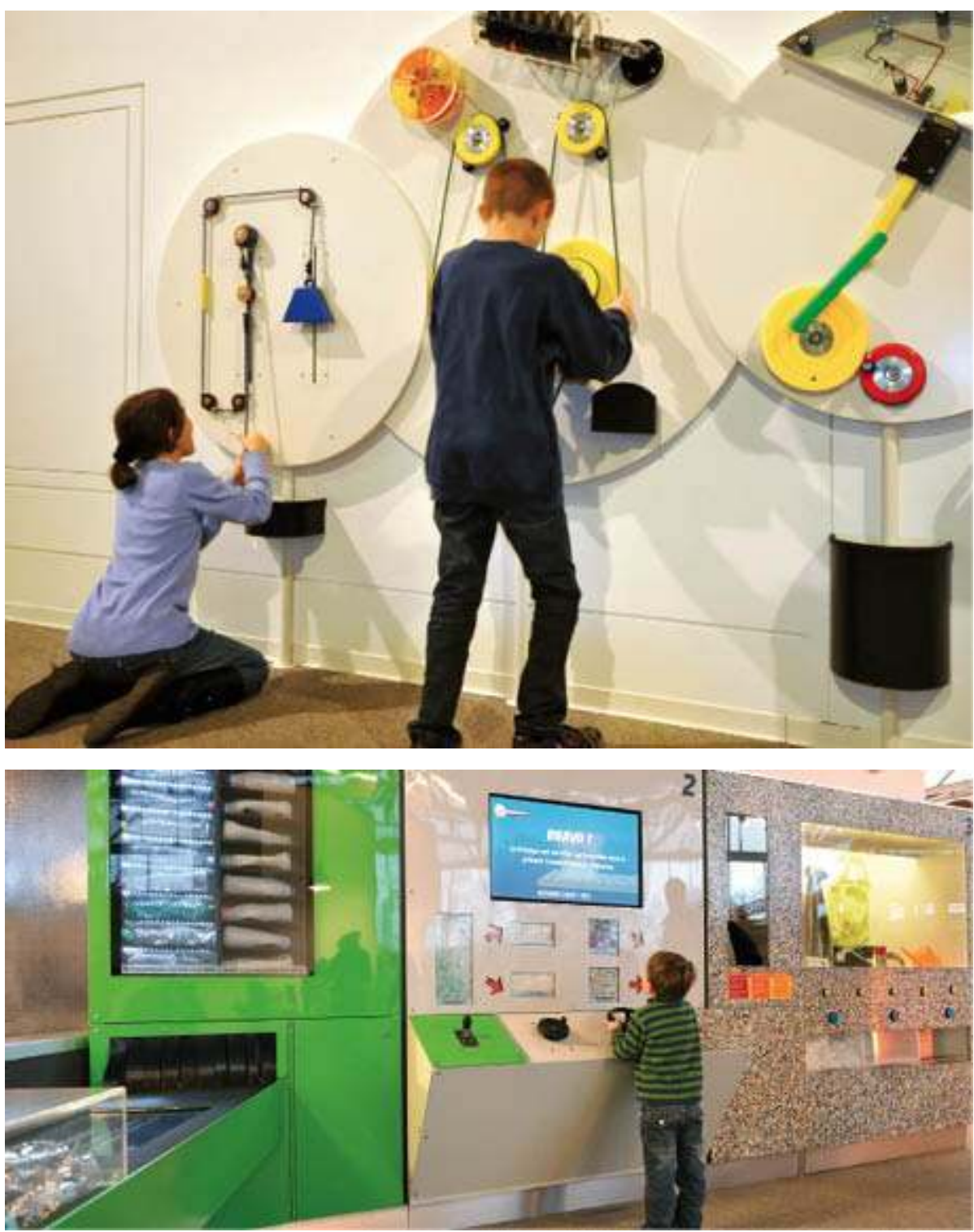

(c) Daniel Schmitt

Dans l'exposition ou au musée, ce n'est cependant pas un auteur ou une personnalité que les visiteurs s'attendent à suivre. C'est le plus souvent une entité plurielle, institutionnelle. L'institution ne désigne nullement ici l'autorité anonyme, sourde et aveugle. Il s'agit plutôt d'une institution au sens anthropologique, une instance construite 
historiquement par de très nombreuses médiations transmises et transformées, qui représente le collectif, avec certes des imperfections, des singularités, une hétérogénéité, des contradictions, mais avec a priori le souci d'un respect pour ce qui a la valeur d'un bien commun collectif (la culture, le savoir, la vie ensemble). De ce fait, les visiteurs sont particulièrement tolérants à la variété des propositions de médiation qui manifestent le fait que l'institution s'adresse à tous, et donne à voir pour tous ce qu'elle propose. Ce respect supposé être partagé fait de l'exposition un endroit où l'on s'en remet avec totale confiance à l'hypothèse que chacun fait au mieux pour l'autre (y compris en tant que visiteur) même si c'est un pari difficile. D'ailleurs il est rare que l'on visite exclusivement pour soi, même si l'on est seul. Le parcours est effectué à la rencontre, avec, pour, un Autrui plus ou moins incarné.

Pour autant, le parcours est un foyer de malentendus possibles, lorsque les visiteurs tentent d'interpréter des éléments qui sont issus de gestes gratuits ou de conflits non réglés lors de la conception. Il n'est pas rare de voir ces conflits se retrouver dans des problèmes d'interprétation lors de la visite, comme par exemple lorsqu'un scénario ne va pas au bout de sa logique.

Il y a donc une série d'efforts de chacun pour faire plusieurs choses à la fois grâce au parcours. Ce qui est agencement dans un espace pour le scénographe est une durée avec un début et une fin pour le visiteur. Ce qui est un discours n'est pas pour autant une parole d'autorité confiée à un individu qui s'exprimerait pour lui-même: il s'agit d'une énonciation plurielle, prise en charge par une diversité d'instances qui cependant se retrouvent toutes, comme les visiteurs, au sein d'un espace institutionnel. Et pourtant, l'espace à la fois physique et symbolique où se situe l'exposition n'est pas ambigu : il assure au contraire le sens général de ce qui est partagé ensemble.

\section{Les visiteurs : qui sont-ils, où vont-ils ?...}

L'attention aux visiteurs se manifeste notamment par les questions que l'on se pose à leurs propos, et avant tout: "qui sont-ils ?". Poser cette question du point de vue de la conception ou de la médiation, ce n'est pas chercher la catégorie socio-démographique à laquelle ils appartiennent ${ }^{3}$. Elle témoigne de situations bien plus familières, celles de la rencontre: " à qui ai-je affaire ?". Cette question en recouvre une nuée d'autres, pas nécessairement très techniques ni très précises, mais qui animent le désir de communiquer : quelles sont leurs visions et leurs modèles de l'exposition? Que viennentils chercher? Que se passe-t-il pour eux ? Pour quoi, avec qui, pour qui visitent-ils ? Où se sentent-ils être ? Existe-t-il une différence entre être visiteur et être membre du public? Quelle place occupe la visite par rapport à toutes leurs pratiques (sociabilité, culture et médias, éducation, auto-formation...)?

De la même manière, on observe que les visiteurs explorent également l'exposition à partir de questions très proches qui animent la visite : "Qu'est-ce qu' ils ont voulu nous faire faire? Pourquoi ont-ils choisi cet objet? Qu'a-t-on voulu faire comprendre? ". Ces questions sont caractéristiques d'une volonté de communiquer, qui elle-même nécessite que les interlocuteurs se sentent être dans un même espace et partager un même temps, au moins sur un plan symbolique. Dans cette perspective, les travaux théoriques en communication ont permis de conceptualiser ce qu'est un média non pas au sens 
empirique de technologie de transmission industriellement développée, mais comme cet espace commun ${ }^{4}$ où circulent des discours.

"Quoi ? Pour qui ? Comment ?" mais également "Où ?". La question du lieu de l'exposition, ce "Où?", si souvent oubliée par les tenants d'un modèle très mécaniste de la communication comme transport d'un message, est de fait capitale pour qu'une rencontre ait lieu ${ }^{5}$.

Des dispositifs multimédia à la Casa del Alabado, musée d'art précolombien à Quito (Équateur).

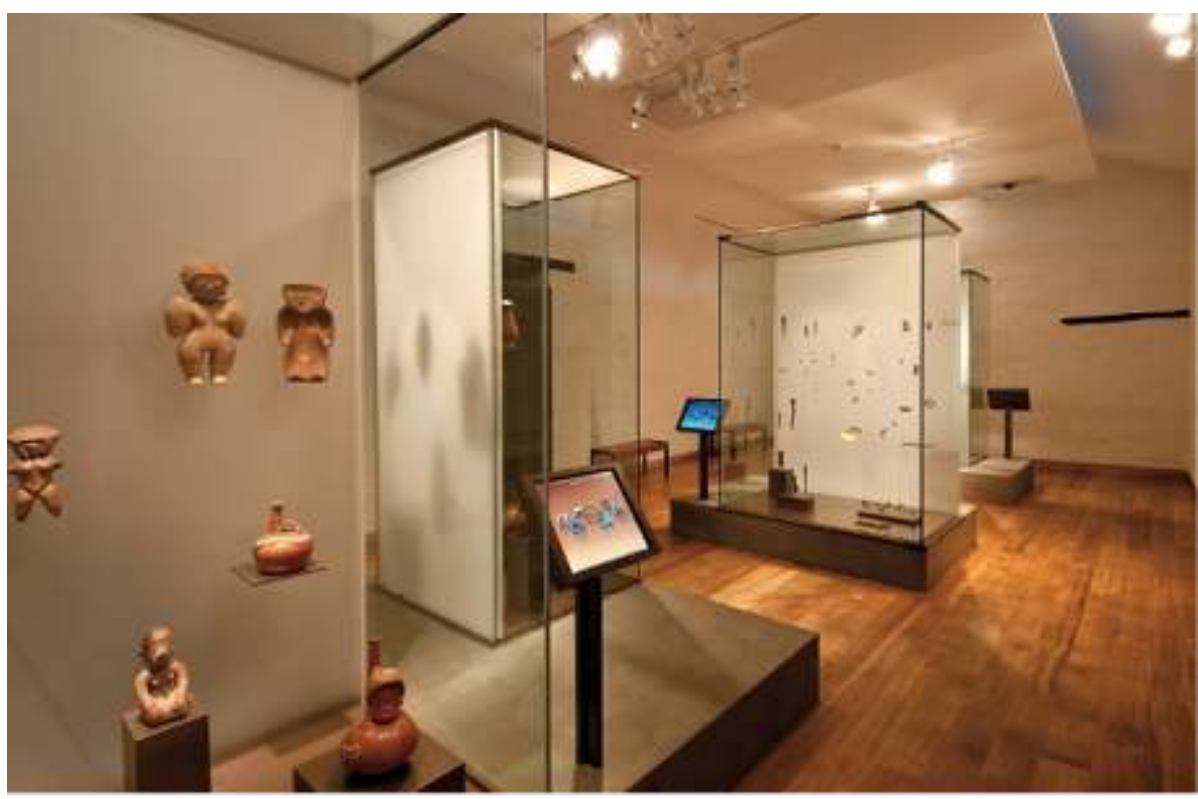

\section{(c) Daniel Schmitt}

Les études de publics montrent que les visiteurs ne perçoivent pas l'exposition comme un produit. Ils se sentent être dans un espace particulier dont on franchit la frontière et dont on peut sortir, parfois indépendamment même de la configuration physique. Par exemple, on peut se sentir "hors de l'exposition" dans un espace partenaire qui est manifestement installé par une instance externe ("est-ce qu'on est encore dans le musée ici ?") comme on peut se sentir dans l'espace muséal en écoutant un commentaire d'audioguide proposé par le musée dans le cadre d'une visite urbaine ${ }^{6}$.

\section{L'économie de visite}

Visiter revient nécessairement à sélectionner dans un ensemble trop riche pour la durée d'une visite d'exposition qui le plus souvent ne pourra pas être renouvelée. Il est donc essentiel d'aider le visiteur à aborder l'exposition, l'accueillir et le guider, en lui rendant manifestes les intentions de médiation dès les premiers moments. Or, les études réalisées montrent que les abords et les entrées des expositions sont parfois les espaces les plus ambigus et les plus brouillés dans le registre de la conception, même dans les expositions les plus soignées. Il arrive en effet que l'entrée soit partagée entre des instances qui n'ont ni travaillé ensemble, ni participé à la réflexion générale sur l'exposition (cela peut être le cas par exemple des services de communication, de partenaires financiers...). Elle s'apparente également parfois à une sorte de recoin, où sont littéralement "casés" tous 
les éléments de contexte, comme dans certaines expositions d'art, obligeant alors les visiteurs à stationner, déjà, au sein d'un espace extrêmement encombré, dans une préface de l'exposition qu'ils n'osent guère sauter par souci de ne rien rater d'important.

De même, les expositions se terminent souvent directement par une sortie abrupte et sans retour. La boutique du musée, intégrée à l'expérience, devient alors le lieu du temps nécessaire pour rester encore, conclure, garder quelque chose ${ }^{7}$.

\section{Culture des médiations, culture médiatiques, frontières}

La médiation dispose rarement d'un espace physique propre dans les musées ou dans l'exposition, même si l'on voit apparaître dans le parcours des salles d'ateliers pédagogiques scénographiés, parfois ouvertes sur l'exposition, dans son prolongement et son enrichissement. Car la médiation contribue très largement à la construction de cet espace institutionnel, qui se déploie en extension et se creuse en profondeur et en lien avec "le monde" dans lequel le visiteur est accompagné et où il s'implique lui-même en tant que témoin, relais (pour ses enfants par exemple ou pour autrui), usager, familier, membre (du public, de l'institution, de la population de référence) ${ }^{8}$...

Parcours au château de Lichtenberg en Alsace

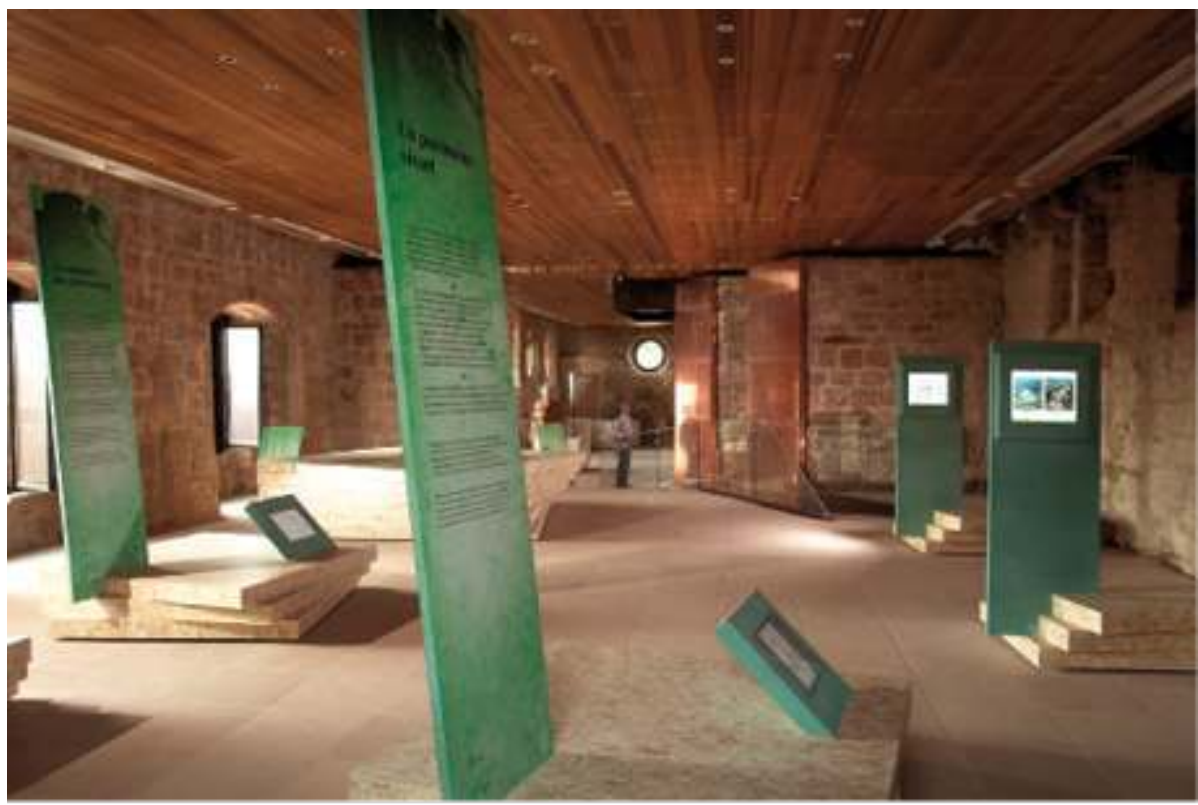

(c) Christian Creutz

La médiation, à travers ses propositions multiples, amplifie et creuse ces parcours dans les "mondes" que créent les expositions et les musées, dans et hors les murs. À l'inverse, au sein même des espaces muséaux, les visiteurs, comme nous l'avons dit, peuvent ressentir physiquement des frontières pourtant peu visibles. La multiplication, dans les expositions, d'interventions totalement autonomes installées par des instances qui défendent des logiques divergentes et qui ne partagent pas l'attention ou la considération pour les visiteurs membres du collectif de référence, sont autant de frontières qui interviennent dans les parcours des visiteurs. 


\section{Parcours, formation et dialogue} attention et dont j'ai voulu rendre compte. Ce parcours incarné fait apparaître un autre type d'espace, symbolique et riche de rencontres potentielles entre les publics et l'ensemble des acteurs de la conception et de la médiation.

27 Je souhaiterais, pour conclure, souligner la découverte qu'a été pour moi chaque formation proposée à l'analyse des parcours d'exposition. Ces formations, toutes différentes, ont été marquées par des moments de dialogue multiples au fil des jours, puis au fil des années, qui ont enrichi cette notion du parcours. Certaines ont fait apparaître des malentendus dont nous ne saurons jamais s'ils étaient irréductibles. D'autres ont, tout au contraire, illustré la réalité d'une construction commune qui, d'une certaine manière, est devenue l'analogue de l'articulation entre programmation, conception, médiation et visite. Située au cœur de l'exposition, ces correspondances en font un espace et un temps qui peuvent s'approcher d'une Eutopie, un lieu non pas mythique et métaphorique mais l'espace bien réel et vivant de la construction culturelle.

\section{NOTES}

1. Ce texte est basé sur la réflexion collective menée avec de nombreux intervenants et participants pour proposer et faire évoluer avec l'équipe de l'OciM les six formations "Comprendre et analyser le parcours de l'exposition" proposées entre 2006 et 2014. Il doit tout particulièrement aux échanges avec Angèle Fourès.

2. Ces outils ne sont pas publiés. Dans les différentes formations de l'OCIM, nous avons ainsi notamment pu voir ceux qui ont été élaborés par Dominique Vitale, Alexandre Delarge, Sophie Grisolia, Hervé Groscarret.

3. Lorsque nous invitons des personnes à participer à un évènement, nous ne cherchons pas à déterminer leur profil pour nous adresser à eux : nous cherchons à travers leur regard, leurs réactions, comment établir un dialogue. Il en est ainsi avec les visiteurs, même si la situation est autrement compliquée puisqu'ils ne sont pas encore là au moment où se font les actions de médiations.

4. Voir les travaux de Jean Davallon, notamment bien sûr L'exposition à l'œuvre. Paris : L'Harmattan, 1999.

5. Sophie Grisolia, conceptrice d'expositions au Muséum national d'Histoire naturelle, lors d'une présentation de sa démarche a ainsi déployé les questions qu'elle se posait lorsqu'elle travaillait à une exposition.

6. Cette différence de perception de l'espace auquel renvoie l'audioguide a été bien étudié de 2003 à 2005 lors de la recherche pour le programme EECOOT, notamment dans le volet mené en 
collaboration par Tout'O Phone (Philippe Charlot), le laboratoire Communication, Culture et Société de l'ENS de Lyon (Sophie Deshayes et Joëlle Le Marec), et l'équipe médiation du musée Gadagne de Lyon (http://c2so.ens-lyon.fr/spip.php?article41).

7. Au Centre d'Histoire de la Résistance et de la Déportation à Lyon, avant sa rénovation, les visiteurs pouvaient repartir avec un exemplaire imprimé de chants de la Résistance. En revanche, ils sortaient en traversant une salle de projection qui rompait totalement, et brutalement, avec l'atmosphère presque intime et dense de l'imprimerie clandestine qui la précédait.

8. Lors d'une recherche auprès des publics des manifestations littéraires en Rhône-Alpes, réalisée en 2009 et 2010 par Roxana Ploestan (École Normale Supérieure de Lyon, équipe Communication, Culture et Société) pour l'Agence Rhône-Alpes pour le Livre et la Documentation, la chercheuse montrait l'existence pour les visiteurs d'un espace symbolique reliant l'ensemble des médiations du livre et de la lecture déployées au moment de la manifestation, sur des sites multiples parfois, en requalifiant les frontières entre espaces de l'échange marchand et espaces des valeurs du livre et de la lecture héritées, transmises et partagées. C'est par la reconnaissance, dans ce même espace, de quantité de pratiques de médiation de la lecture autre que la lecture assidue (conférences, rencontres, visites...) que les visiteurs éprouvaient la légitimité assumée de nouvelles pratiques du livre, ré-institutionnalisées par la manifestation, et surtout, par la magie des parcours et déambulations des habitants dans le temps et l'espace de cette manifestation devenue exposition du "monde" de la lecture.

\section{RÉSUMÉS}

Cette réflexion sur le parcours de visite d'un musée ou d'une exposition souligne qu'au-delà de la simple relation entre l'offre et la demande, le parcours est un dialogue et un lieu d'échange entre les scénographes, les muséologues, les médiateurs et les visiteurs, pour lesquels il n'a pas la même signification ni le même objectif.

\section{INDEX}

Mots-clés : numérique, web

\section{AUTEUR \\ JOËLLE LE MAREC}

professeur à l'université Paris 7 Diderot

membre du Centre d'études et de recherches interdisciplinaire sur les Lettres, Arts et Cinéma

(CERILAC)

directrice du master Journalisme, Culture et Communication scientifique 